\title{
Examining the Association between Polish Migrant Status and Health Preferences Using a Novel Application of a Smaller Design EQ-5D-5L Valuation Study
}

\author{
Dan Kelleher ${ }^{5}$ (1) Samer Kharroubi ${ }^{2} \cdot$ Edel Doherty $^{1} \cdot$ Gianluca Baio $^{3} \cdot$ Ciaran O'Neill $^{4}$
}

Accepted: 21 November 2021 / Published online: 26 January 2022

(c) The Author(s) 2022

\begin{abstract}
Introduction Migrants have different utilisation of healthcare services and health-related behaviours than host populations. A potential factor that may contribute to the notable differences in healthcare use and health-related behaviours between migrants and host populations is how these groups value health. Those who place a high value on health have greater healthcare-seeking practices than those who do not.

Objective The aim of this study was to examine how Polish migrants and native Irish differ in health state utility valuations using a novel application of a smaller design EQ-5D-5L valuation study.

Methods This study uses health preferences as a predictor of how one values health. We examined the EQ-5D-5L health preferences of 119 Polish migrants and 123 native Irish, both residing full-time in Ireland. To do so, we used a novel application of a smaller design EQ-5D-5L valuation study that consisted of 30 health states and a targeted sampling strategy coupled with a Bayesian statistical nonparametric model. We collected data from June 2018 to September 2019.

Results Our results highlight that Polish migrants and native Irish differ in their health preferences for and valuation of severe health states. Polish migrants place meaningfully higher utility valuations of 0.1 or more on the three most severe health states compared with the native Irish.

Conclusion This study can provide an understanding of a potential new factor underpinning some of the disparities in healthcare utilisation and health-related behaviours among migrants and host populations in Europe. This study also provides proof of principle for using a smaller design EQ-5D-5L valuation study to explore differences in health preferences among other minority subgroups, which can otherwise be hard to uncover when using the secondary analysis of national EQ-5D-5L valuation studies.
\end{abstract}

\section{Key Points for Decision Makers}

Polish migrants apply a higher utility valuation to more severe health states than native Irish respondents.

Differences in health preferences potentially infer differences in health-related behaviours between migrants and natives.

Smaller design EQ-5-D-5L valuation studies can be used in examining the health preferences of hard-to-research subgroups.

Dan Kelleher

d.kelleher2@nuigalway.ie; dankelleher92@gmail.com

Extended author information available on the last page of the article

\section{Introduction}

European countries have become increasingly popular destinations for migrants from other European states as well as migrants from outside of Europe. The European Union (EU) has a diverse stock of migrant populations. Migrants make up an estimated $13.2 \%$ of the EU's total population, with individuals born in another EU member state totalling $5.5 \%$ of the population and those born in a non-EU country making up $7.7 \%$ of the population [1]. Numerous studies have examined the healthcare service use and health-related behaviours of migrants relative to host populations in Europe [2-4]. In Europe, migrants are noted to have different utilisation of healthcare services than host populations, with the differences most evident in preventive healthcare uptake, as migrants consistently have lower uptake than host populations [5-7]. Migrants are also shown to have different health-related behaviours 
than host populations, and have been noted to engage in more unhealthy behaviours when compared with host populations in Europe, such as increased smoking and alcohol consumption [8].

Marked differences and inequalities in the healthcare utilisation and health-related behaviours of migrants and host populations in Europe have been identified in the literature. Understanding the many factors that drive these disparities is pivotal for designing, adapting, and implementing a migrant health policy in Europe, and a multitude of studies have examined such factors, such as the formal and informal barriers to healthcare faced by migrants, transnationalism and cultural attitudes and beliefs regarding health and healthcare held by migrants [9-13]. A potential factor that may contribute to some of the notable differences in the healthcare use and health-related behaviours of migrants compared with host populations is how these groups value health.

An individual's valuation of health has been shown to help determine their use of healthcare and health-related behaviours, as individuals who place a high value on health have greater healthcare-seeking practices, especially preventive care use, than those who do not [14-16]. Determining how migrants and host populations differ in their valuation of health is an under-researched area despite the potential insight this may provide into the noted differences between groups in their healthcare use and health-related behaviours. Health preferences are a predictor of an individual's valuation of health. Health preferences as a variable, proxies the value an individual places on health (such as how an individual values different health state utilities), which can likely inform an individual's healthcare use and healthrelated behaviours $[17,18]$.

In this study, we examine the association between Polish migrant status and health preferences as measured by the EQ-5D-5L health descriptive system. More specifically, we examine the health preferences of Polish migrants residing full-time in Ireland relative to a group of native Irish also residing full-time in Ireland. The Polish migrant group are the largest migrant group residing in Ireland [19]. As such, understanding how Polish migrants and native Irish differ in their health preferences, and ultimately in their valuation of health, may provide new insight into the reasoning that underpins some of the disparities in healthcare use and health-related behaviours between migrants and host populations in Europe.

\section{Methods}

The usual manner in which to conduct any subgroup analysis of health preferences, such as comparing migrants and a host population, is to use a secondary analysis of national EQ5D-5L valuation studies. Numerous countries have carried out national valuation studies [20-23], and other valuation studies have emerged that use novel study design methods to examine EQ-5D-5L health preferences [24]. National valuation studies not only primarily provide researchers with data on health preferences to inform cost-utility analysis (CUA) but they also provide researchers with a potential opportunity to compare the health preferences of various subgroups. However, one issue is that national valuation studies are not primarily designed to examine differences in health preferences between subgroups and may not be powered to detect such differences.

When using a secondary analysis of national valuation studies to compare subgroup health preferences, an issue can be the relative sample size of the subgroups uncovered in the overall sampling of the general public [17, 18, 25-27]. Solutions to this issue have included oversampling of specific subgroups alongside national valuation studies, or, as recommended by Sculpher and Gafni [28], conducting an additional survey to uncover subgroups in greater detail. The authors suggest this additional survey can complement national valuation studies and would allow for the targeted sampling of subgroups. This targeted approach requires a novel application of EQ-5D-5L valuation exercises whereby a reduced sample size values a smaller number of health states compared with national valuation studies [29, 30]. Ultimately, this requires a smaller design valuation study, which can be deployed to target subgroups for whom preferences are thought to differ, such as migrants and host populations.

\subsection{Survey Design and Sampling Strategy}

To capture data on health preferences, we estimated preferences for the dimensions of the EQ-5D-5L using the composite time trade-off (cTTO) approach [31]. cTTO data were collected for a group of Polish migrants and native Irish, both residing full-time in Ireland. Polish migrants were selected for the analysis as they are the largest nonIrish population living in Ireland, with over 120,000 Polish migrants residing in Ireland [19]. The majority of Polish migrants in Ireland are aged between 30 and 40 years, making up $47 \%$ of the Polish population in Ireland, with $50.49 \%$ males and 49.51 females [19]. Each respondent valued six practice states plus one block of 11 cTTO states (giving 11 observations per respondent), which all respondents completed in English. A sample size of 240 (120 Polish migrants and 120 native Irish) was deemed appropriate as it was the maximum that could be collected based on the resources available for this research. Data were collected from June 2018 to September 2019, with the EuroQol Research Foundation providing the orthogonal design of health states for valuation. All interviews were collected using the EuroQol Research Foundation's 
EuroQol-Portable Valuation Technology (EQ-PVT), which is a computer-assisted personal interview (CAPI) software and protocol [30,32]. Ethical approval for the study was granted by NUI Galway's Research Ethics Committee (application number 18-Mar-13).

\subsection{Experimental Design}

We used a similar orthogonal design as Yang et al. [30], which, in this study, entailed of 25 health states, including the 55555 'pits' state plus five mild health states to compensate for an underrepresentation of mild states, for a total of $30 \mathrm{EQ}-5 \mathrm{D}-5 \mathrm{~L}$ health states to be valued. Using an orthogonal design allows us to value a smaller number of health states and use a smaller sample size compared with that of a full national valuation study, which uses 86 cTTO health states and a minimum of 1000 respondents [22]. Yang et al. [29, 30] have examined and compared estimates of the smaller design EQ-5D-5L valuation study with the traditional EQ-5D-5L valuation study of 86 health states. Both papers note that the smaller design preforms well compared with the larger design and does not lead to significant increases in prediction errors when modelling cTTO data.

\subsection{Blocking}

The 30 health states were divided into three blocks of 11 states using the blocking algorithm included in the "AlgDesign" package in R. The blocking algorithm divides the states over the blocks to ensure that within-block variance is maximised so that observations on the full utility range is achieved.

\subsection{Interviewers and Respondents}

We collected cTTO state valuations for the three blocks of health states for Polish migrants who were born in Poland and residing full-time in Ireland and a group of native Irish who were born in Ireland and residing full-time in Ireland. Respondents were asked before the survey began to state which country they were born in and whether they live in Ireland full-time. Seven interviewers and one study coordinator collected the data. Respondents were recruited into the study through a Facebook study page, emailing the study coordinator, or being contacted through friends and family using snowball sampling. Respondents provided written consent to participate in the study.

The extended survey design and sampling strategy can be seen in more detail in the electronic supplementary material.

\subsection{The Nonparametric Modelling Approach}

Health state utility valuation data are known to have a complex data structure that can be truncated, skewed and suffer from heteroscedasticity [33]. A nonparametric method allows the utility function to take any form, employing Bayesian hierarchical modelling. This approach can represent complex data, such as health state utility valuation, well and can compare multiple subgroups with small sample sizes as in this study, hence why this method was used for this analysis [34]. We used a similar Bayesian statistical nonparametric model as Kharroubi et al. [35]. Kharroubi et al. [36] have documented how this approach provides a more flexible inference for preference functions when compared with parametric methods.

The modelling aims to estimate the differences between the Polish migrant group and the native Irish group by estimating a general model that explains health state utility valuations in terms of the EQ-5D-5L health descriptive system, respondent background characteristics and migrant status. For $j=1,2, m$, let $n_{j}$ be the number of health states that were valued by the jth respondent. An EQ-5D-5L health state is a vector of five numbers, which is represented by the symbol $x$. For $i=1,2, \ldots, n_{j}$ and $j=1,2, \ldots, m$, let $x_{i j}$ be the $i$ th health state valued by respondent $j$ and let $y_{i j}$ be the cTTO utility valuation given by respondent $j$ for that health state. Finally, $h\left(z_{j}\right)$ is the vector of covariates for respondent $j$ that includes Polish migrant status ( $1=$ Polish migrant; $0=$ native Irish), sex $(1=$ male; $0=$ female $)$, and age.

The $i$ th valuation by respondent $j$ is modelled as shown in Eq. 1:

$y_{i j}=1-\exp \left(\gamma^{\prime} h\left(z_{j}\right)+\alpha_{j}\right)\left\{1-u_{c}\left(x_{i j}\right)\right\}+\varepsilon_{i j}$,

where $h\left(z_{j}\right)$ is a vector of functions of the covariates $z_{j}$ and $\gamma$ is a vector of parameters that, combined, determine the effect of the covariates on the respondents' valuations; $\alpha_{j}$ is a zero-mean random respondent effect; $\varepsilon_{i j}$ is a zero-mean random error; $u_{c}(x)$ is a function of the health state vector $\mathrm{x}$; and the subscript $\mathrm{c}$ represents the respondent's nationality. The interpretation of $u_{c}(x)$ is as a base utility function; if the population expectation of $\exp \left(\gamma^{\prime} h\left(z_{j}\right)+\alpha_{j}\right)$ is 1 , then $u_{c}(x)$ is the population utility measure, where $c=1$ if respondent $j$ is a Polish migrant and $c=0$ if the respondent is native Irish.

In Eq. 1, every respondent values perfect health as having a utility valuation of 1 (subject to measurement error $\varepsilon_{i j}$ ), no matter what that respondent's covariates are and regardless of the random effect $\alpha_{j}$. The covariates and random effects enter model (1) multiplicatively. As a result, they have greater effect the further the base utility is from 1 . This implies that we are likely to see more variability in the respondents' valuations for severe health states than for mild states. 
Normal distributions are assigned for the random terms as shown in Eq. 2:

$\alpha_{j} \sim N\left(\gamma^{\prime} h\left(z_{\mathrm{j}}\right), \tau^{2}\right)$ and $\varepsilon_{i j} \sim \mathrm{N}\left(\theta, v^{2}\right)$,

where $\tau^{2}$ and $v^{2}$ are parameters to be estimated. The distribution of the respondent effect $\lambda_{j}=\exp \left(\gamma^{\prime} h\left(z_{j}\right)+\alpha_{j}\right)$ is then log-normal, representing a skewness that is often observed in health state valuation data. The $z$ 's are centred to ensure that they have zero means, and hence the value of $z_{j}$ for a typical person is 1 .

The base utility functions for each group are provided below $\left(u_{0}(x)=\right.$ Irish; $u_{1}(x)=$ Polish $)$. The relationship between the two base utility functions is modelled as shown in Eqs. 3 and 4.

$u_{0}(x)=\mu_{0}+\beta_{0}^{\prime} x+d(x)$,

$u_{1}(x)=\left(\mu_{0}+\mu_{1}\right)=\left(\beta_{0}^{\prime}+\beta_{1}^{\prime}\right) x+d(x)$,

The expression $\mu_{0}+\beta_{0}{ }^{\prime} x$ in Eq. 3 states that the underlying utility function $u_{0}(x)$ for the Irish respondents will usually behave like a simple linear combination of the elements of the health state description vector $x$. The coefficients $\beta$ ${ }_{0}$ (which are expected to be negative) represent the rate at which utility generally declines when we increase the severity level in the corresponding dimension of $x$. The comparable expression in Eq. 4 modifies these underlying trend variables with additional coefficients $\beta_{1}$ to reflect dimensionspecific differences between the Polish migrants and native Irish.

The term $d(x)$ represents a deviation from the simple linear trend that is common to the Polish migrants and native Irish respondents. We treat $d(x)$ as an unknown function, and therefore in a Bayesian nonparametric framework, it becomes a random variable. It has a zero mean and constant variance and is constrained by a correlation between $d(x)$ and $d\left(x^{\prime}\right)$ for two different states $x$ and $x^{\prime}$ that decreases as the distance between $\mathrm{x}$ and $x^{\prime}$ increases. The effect of this is to assert that if $x$ and $x^{\prime}$ describe very similar health states, their utilities will be approximately the same. This will allow the preference function to vary smoothly as the health state changes. This model allows $d(x)$ to take any value reflecting a nonparametric specification, and hence the utility functions are not constrained. It is in this regard that we note our model as nonparametric, similar to Kharroubi et al. [35].

It is important to note that the population utility function, is not, in general, the base utility function $u_{c}(x)$. The base utility function is the utility function for a person whose respondent effect is 1 . This will be the base utility function only if the mean or median respondent effect across the population is 1 . Otherwise, the population utility function in country $c$ is as shown in Eq. 5: $\bar{u}_{\mathrm{c}}(x)=1-\bar{\alpha}_{\mathrm{c}}\left(1-u_{\mathrm{c}}(x)\right)$,

where $\bar{\alpha}_{\mathrm{c}}$ is the population mean/median respondent effect for that country and $u_{\mathrm{c}}(x)$ is the corresponding base utility function. The fact that, in general, $\bar{\alpha}_{\mathrm{c}} \neq 1$ and the population utility is not the base utility affects how we interpret the utility slope parameters $\beta_{0}$ for the native Irish and $\left(\beta_{0}+\beta\right.$ $\left.{ }_{1}\right)$ the Polish migrants. The change in utility for an increase of one level in a particular dimension is represented as an underlying trend, not by the corresponding slope parameter but by the parameter multiplied by $\bar{\alpha}_{\mathrm{c}}$.

In this paper, we only consider the Bayesian statistical nonparametric model to estimate the differences between both groups, as the validity of the approach has been established in the above-cited papers $[35,36]$. It is also worth noting we are not interested in estimating a value set from the responses collected but only to compare health state utility valuations. The models were estimated in MATLAB.

\section{Results}

A total of 242 survey interviews were completed (119 Polish migrants and 123 native Irish). All interviews adhered to the EuroQol protocol [37] and quality control checks ensuring all interviews were deemed to be of high quality with no interviews being dropped from the analysis. Table 1 reports the descriptive statistics of the study and how each variable was described in the analysis.

In Table 2, the observed mean utility values, posterior inference (predicted) mean utility values and standard deviations are reported for both the Polish migrant and native Irish groups for the health states valued. The observed mean cTTO utility valuations ranged from -0.57 (55555) to 0.96 (11211) for the Polish migrant group, and from -0.61 (55555) to 0.96 (11211) for the native Irish group. The predicted mean cTTO utility valuations ranged from -0.42 (55555) to 0.94 (11211) for the Polish migrant group, and from -0.59 (55555) to 0.99 (11211) for the native Irish group.

For the purpose of interpreting the results, the health states are organised by their misery score, which is a proxy measure of the severity of the health state that is calculated by adding together the levels of each health state (for example, health state (33252): $3+3+2+5+2=15$ ) [38]. For this analysis, health states with a misery score of 10 or less are considered mild, health states with a misery score of $>10$ and $\leq 15$ are considered intermediate, and health states with a misery score $>15$ are considered severe.

The Polish migrants' predicted mean utility valuations were lower for mildstates compared with the native Irish. For example, for state 11121, the predicted values for the Polish migrant group is 0.9 , and 0.92 for the native Irish 
Table 1 Sample descriptive statistics

\begin{tabular}{lll}
\hline Variable & $\begin{array}{l}\text { Polish migrant } \\
{[n=119 ; 49 \%]}\end{array}$ & $\begin{array}{l}\text { Native Irish } \\
{[n=123 ;} \\
51 \%]\end{array}$ \\
\hline Age, years [continuous] & $38(7.6)$ & $34(15.1)$ \\
Sex [1 = male] & $22 \%(0.41)$ & $55 \%(0.5)$ \\
Married [1 = married] & $69 \%(0.46)$ & $27 \%(0.44)$ \\
Urban household [1 = urban] & $76 \%(0.43)$ & $43 \%(0.5)$ \\
$\begin{array}{l}\text { Number of dependents <18 [continu- } \\
\text { ous] }\end{array}$ & $1.14(1.04)$ & $0.35(0.78)$ \\
$\begin{array}{l}\text { Employed [1 = employed] } \\
\text { Private health insurance [1 = yes] }\end{array}$ & $76 \%(0.42)$ & $54 \%(0.5)$ \\
$\begin{array}{l}\text { Third-level education [1 = yes] } \\
\text { Self-rated health using EQ-5D-5L } \\
\text { misery score [continuous] }\end{array}$ & $76 \%(0.43)$ & $56 \%(0.5)$ \\
$\quad \begin{array}{l}\text { Self-rated health using VAS [con- } \\
\text { tinuous] }\end{array}$ & $84(11.66)$ & $5.6(1.09)$ \\
\hline
\end{tabular}

Standard deviation is reported in parentheses

VAS visual analogue scale

group. In the intermediate states, Polish migrants' predicted mean utility valuations are higher on more states than the native Irish. However, the native Irish are still predicted to place a higher valuation on some intermediate states, with no apparent trend present. For example, for state 11453, the predicted valuation is higher for the Polish migrant group at 0.08 , and -0.02 for the native Irish group. For state 13541, the predicted valuation is lower for the Polish migrant group at 0.14 , and 0.16 for the native Irish group. A trend emerges in the severe states, with Polish migrants' predicted mean utility valuations being higher on the severe states (except one state) compared with the native Irish. For example, for state 34444, the predicted value for the Polish migrant group is -0.2 , and -0.3 for the native Irish group. The predicted mean valuations show that Polish migrants valued seven states below 0 (i.e. worse than dead) and the native Irish valued 11 states below 0 .

The covariates and random effects enter the Bayesian model multiplicatively, unlike the traditional regression model which applies additively $[35,36]$. As a result, they have more effect the further the base utility is from 1 . This implies that we will see greater variability in the respondents' valuations for poor health states than for good states. This is indeed a feature that is observed in valuation data and is one way in which our model better represents reality than that of conventional classical regression models. We also believe that our modelling is more realistic and allows a clear separation between the overall tendency for Polish respondents to give higher valuations (but with greater differentials for worse health states) from the way in which the Polish population differs from the Irish in its reaction to different health dimensions.

Henry et al. [39] and Shaw et al. [26] both note that a minimally important difference of $\geq 0.1$ is a meaningful difference in EQ-5D health state utility valuations. From this analysis, a difference of $\geq 0.1$ is found in seven health states between the Polish migrants and native Irish, namely states $31125,11453,22245,14335,42354,34444$ and 55555. Polish migrants are also predicted to place higher utility valuations on the three most severe health states (42354, 34444 and 55555) where meaningful differences $>0.1$ are found.

Figure 1 shows the observed versus predicted mean utility values for the Polish migrant group and Fig. 2 shows the same for the native Irish group. The yellow line in each graph represents the residuals obtained between the observed valuation and the predicted valuation for each group. As can be seen from the respective graphs, the valuations for the Polish migrant group are very similar for mild and intermediate states, with discrepancies emerging for severe states, while the inverse is true for the native Irish valuations. Figure 3 shows the histograms of the conditional posterior distributions of the covariate effects (sex, age and Polish migrant). The histogram of the conditional posterior distribution of the Polish migrant covariate demonstrates it to have an effect as the distribution is centred further away from zero. The result is similar for the age covariate. The effect is weaker for the sex covariate as the distribution is seen to be more closely centred over zero. Both Bayesian statistical nonparametric models achieved a low root mean square error (RMSE) of 0.06 and 0.08 for the Polish migrants and native Irish, respectively. A graph further showing the differences in the predicted utility valuations of both groups can be seen in Fig. 4. The graph shows that differences in predicted utility valuations increases between both groups as the health states increase in severity.

\section{Discussion}

In this study, we examined the association between Polish migrant status and EQ-5D-5L health preferences. Our study is the first to explicitly capture and compare the health preferences of migrants and a host population using a novel application of a smaller design EQ-5D-5L valuation study and targeted sampling strategy. More specifically, this study examined the health preferences of Polish migrants and native Irish, both residing full-time in Ireland. We used the EQ-PVT data collection software and a Bayesian statistical nonparametric model to estimate 
Table 2 Inference for all health states by migrant status

\begin{tabular}{|c|c|c|c|c|c|c|c|c|c|}
\hline \multirow{2}{*}{$\begin{array}{l}\text { Health } \\
\text { state } \\
\text { number }\end{array}$} & \multirow{2}{*}{$\begin{array}{l}\text { Health } \\
\text { state } \\
\text { profile }\end{array}$} & \multirow[t]{2}{*}{ Misery score } & \multicolumn{3}{|c|}{ Polish migrant } & \multicolumn{3}{|c|}{ Native Irish } & \multirow{2}{*}{$\begin{array}{l}\text { Predicted } \\
\text { difference } \\
\text { (Polish-Irish) }\end{array}$} \\
\hline & & & $\begin{array}{l}\text { Observed } \\
\text { mean }\end{array}$ & $\begin{array}{l}\text { Posterior } \\
\text { inference } \\
\text { mean }\end{array}$ & $\begin{array}{l}\text { Standard } \\
\text { deviation }\end{array}$ & $\begin{array}{l}\text { Observed } \\
\text { mean }\end{array}$ & $\begin{array}{l}\text { Posterior } \\
\text { inference } \\
\text { mean }\end{array}$ & $\begin{array}{l}\text { Standard } \\
\text { deviation }\end{array}$ & \\
\hline 1 & 11111 & 5 & 1 & 1 & 0 & 1 & 1 & 0 & 0 \\
\hline 2 & 11112 & 6 & 0.9269 & 0.8941 & 0.037 & 0.9122 & 0.901 & 0.0329 & -0.007 \\
\hline 3 & 11121 & 6 & 0.9137 & 0.9 & 0.0404 & 0.9549 & 0.9197 & 0.0373 & -0.020 \\
\hline 4 & 11211 & 6 & 0.9577 & 0.9363 & 0.0384 & 0.9622 & 0.9883 & 0.0339 & -0.052 \\
\hline 5 & 12111 & 6 & 0.9062 & 0.8816 & 0.037 & 0.9463 & 0.9347 & 0.032 & -0.053 \\
\hline 6 & 21111 & 6 & 0.9025 & 0.9023 & 0.0389 & 0.9585 & 0.9557 & 0.0342 & -0.053 \\
\hline 7 & 12112 & 7 & 0.8262 & 0.8255 & 0.037 & 0.85 & 0.8358 & 0.0372 & -0.010 \\
\hline 8 & 41231 & 11 & 0.6763 & 0.6461 & 0.0511 & 0.7085 & 0.653 & 0.0511 & -0.007 \\
\hline 9 & 31125 & 12 & 0.2587 & 0.2065 & 0.068 & 0.2354 & 0.0923 & 0.0703 & 0.114 \\
\hline 10 & 21514 & 13 & 0.29 & 0.2351 & 0.0644 & 0.2915 & 0.205 & 0.06 & 0.030 \\
\hline 11 & 23323 & 13 & 0.6308 & 0.5773 & 0.0559 & 0.5805 & 0.5264 & 0.056 & 0.051 \\
\hline 12 & 24151 & 13 & -0.0897 & -0.0586 & 0.0779 & -0.0207 & -0.0703 & 0.0743 & 0.012 \\
\hline 13 & 35311 & 13 & 0.5662 & 0.5134 & 0.0539 & 0.6646 & 0.592 & 0.0513 & -0.079 \\
\hline 14 & 11453 & 14 & 0.0812 & 0.0839 & 0.0757 & 0.1598 & -0.0239 & 0.0802 & 0.108 \\
\hline 15 & 13541 & 14 & 0.2037 & 0.1421 & 0.07 & 0.2024 & 0.1553 & 0.0637 & -0.013 \\
\hline 16 & 15224 & 14 & 0.2513 & 0.1464 & 0.0733 & 0.2024 & 0.0775 & 0.0719 & 0.069 \\
\hline 17 & 52421 & 14 & 0.4575 & 0.4328 & 0.0563 & 0.5805 & 0.4911 & 0.053 & -0.058 \\
\hline 18 & 22245 & 15 & -0.0167 & 0.0033 & 0.0827 & -0.1171 & -0.1633 & 0.0881 & 0.167 \\
\hline 19 & 33252 & 15 & 0.1 & 0.0651 & 0.0705 & 0.1467 & -0.0007 & 0.0737 & 0.066 \\
\hline 20 & 51342 & 15 & 0.2256 & 0.1611 & 0.0713 & 0.139 & 0.1268 & 0.0671 & 0.034 \\
\hline 21 & 54213 & 15 & 0.1987 & 0.1855 & 0.0663 & 0.2549 & 0.1964 & 0.0623 & -0.011 \\
\hline 22 & 14335 & 16 & 0.0075 & -0.0431 & 0.0826 & -0.0671 & -0.1748 & 0.0861 & 0.132 \\
\hline 23 & 25432 & 16 & 0.1949 & 0.1387 & 0.0726 & 0.2073 & 0.1335 & 0.0676 & 0.005 \\
\hline 24 & 32533 & 16 & 0.4359 & 0.366 & 0.0658 & 0.3768 & 0.3024 & 0.0669 & 0.064 \\
\hline 25 & 53134 & 16 & 0.1562 & 0.0841 & 0.0778 & 0.1598 & -0.0112 & 0.0808 & 0.095 \\
\hline 26 & 43415 & 17 & -0.0387 & -0.0235 & 0.0766 & 0.0134 & -0.0858 & 0.0754 & 0.062 \\
\hline 27 & 44522 & 17 & 0.2913 & 0.2453 & 0.0674 & 0.3268 & 0.2685 & 0.0612 & -0.023 \\
\hline 28 & 45143 & 17 & 0.0775 & -0.0669 & 0.0883 & -0.1451 & -0.1409 & 0.083 & 0.074 \\
\hline 29 & 42354 & 18 & -0.1313 & -0.1925 & 0.0926 & -0.3293 & -0.3291 & 0.0934 & 0.137 \\
\hline 30 & 34444 & 19 & -0.2167 & -0.1976 & 0.0926 & -0.3463 & -0.3025 & 0.0908 & 0.105 \\
\hline 31 & 55555 & 25 & -0.5723 & -0.4235 & 0.1075 & -0.6053 & -0.5927 & 0.113 & 0.169 \\
\hline
\end{tabular}

Health state 11111 was not valued in the study but was included in the analysis. SD relates to the predicted values

the differences in health state utility valuations between the Polish migrants and native Irish.

Preferences for health are noted to differ due to geographic variation [40] and the results from this study show that migrant status can also lead to differences. In this study, the differences between both groups are most apparent in the severe health states, where Polish migrants are predicted to place higher utility values on these states than the native Irish on average. Polish migrants are predicted to place higher utility valuations on 18 of the 30 health states than the native Irish. Of these, Polish migrants are predicted to place meaningfully higher utility valuations of $\geq 0.1$ on seven health states, including the three most severe health states. It is justifiable to note that where we find differences of 0.1 or larger in health state utility valuations, these differences can be considered meaningful differences [26, 39]. Of the remaining 12 states, the native Irish are predicted to place higher utility valuations on them when compared with the Polish migrants. However, for each state, the difference is $<0.1$ and is therefore not considered meaningful. The largest predicted difference between both groups was in their respective utility valuations of the 55555 state, whereby Polish migrants place a higher utility valuation of 0.17 relative to the native Irish.

The differences in the utility valuations of the health states may be linked to the Polish migrants and native Irish 


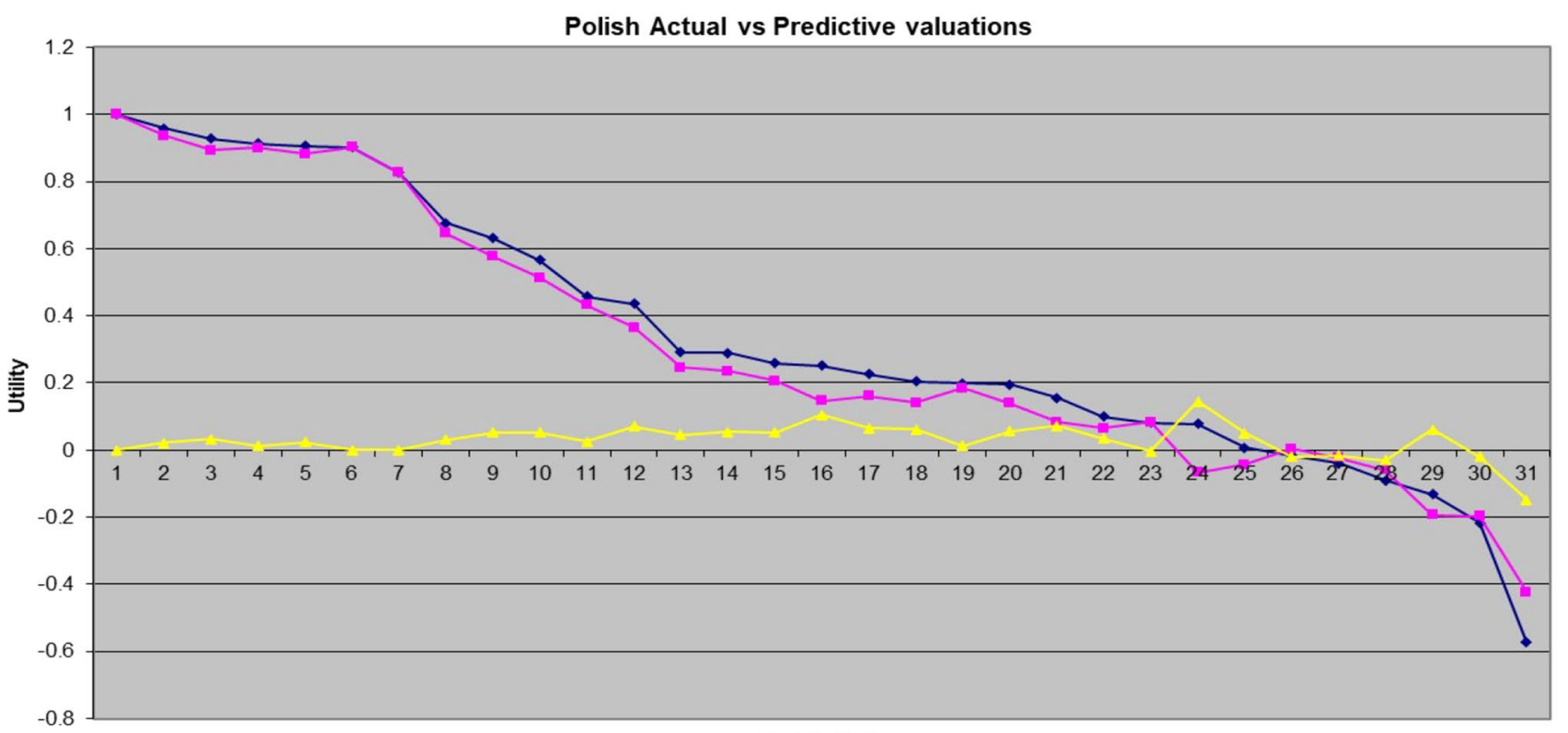

Health State

$\rightarrow$ Observed Mean $\rightarrow-$ Predicted Mean $\rightarrow$ Residuals

Fig. 1 Polish migrant observed vs. predicted valuations

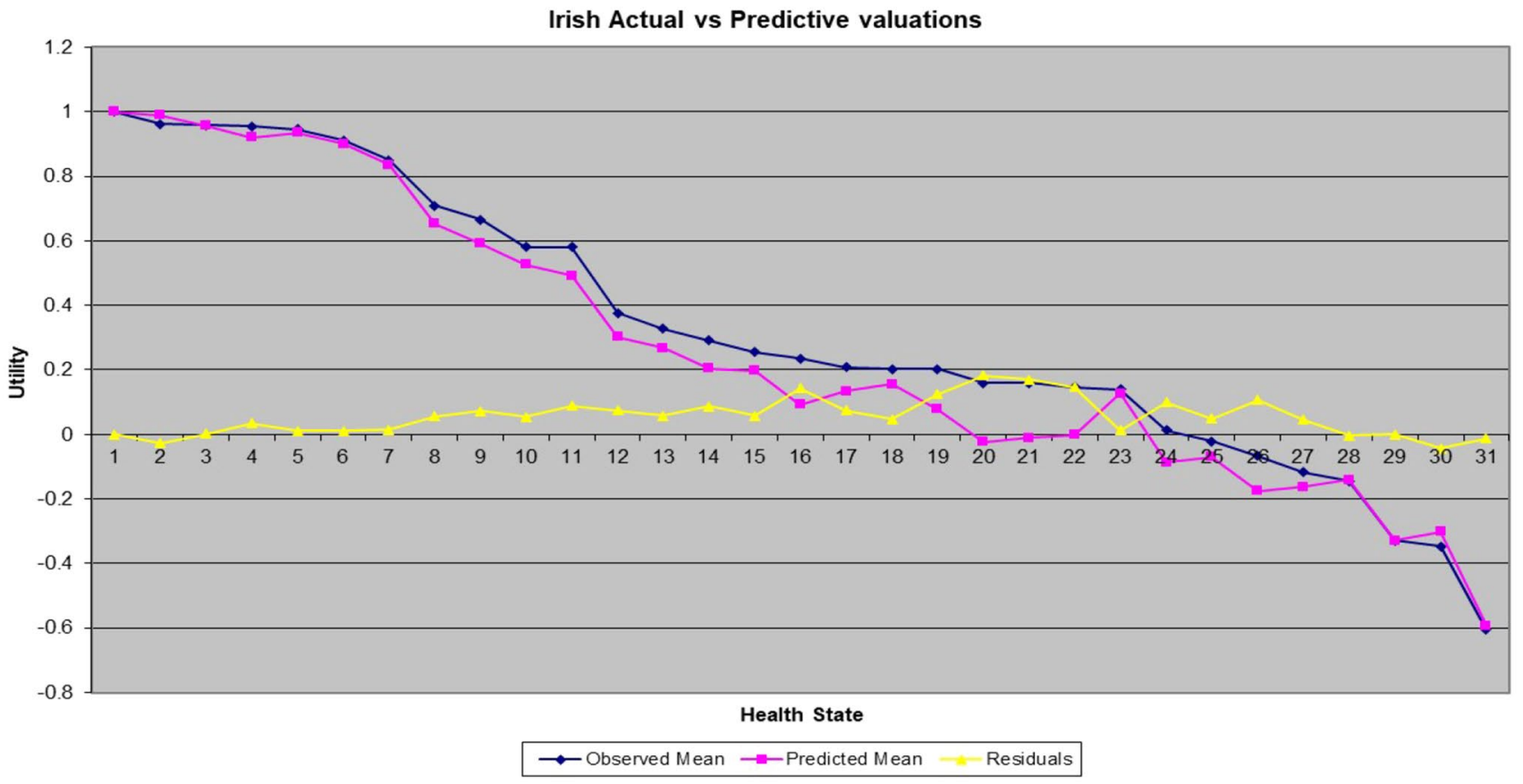

Fig. 2 Native Irish observed vs. predicted valuations

having different rates of time preference (discount rate). cTTO utility valuations are underpinned by an individual's rate of time preference, with an individual who places a high utility valuation on health states currently argued to have a lower rate of time preference (i.e. discount future health less heavily) than those who place a low valuation on it [18, 26]. The larger differences in the predicted valuations of the more severe health states compared with the milder states is somewhat to be expected due to the covariates and random effects entering the model multiplicatively, as noted 
Fig. 3 Histograms of the conditional posterior distributions of the covariate effects
Fig. 4 Predicted differences in cTTO utility valuations by group. cTTO composite time trade-off
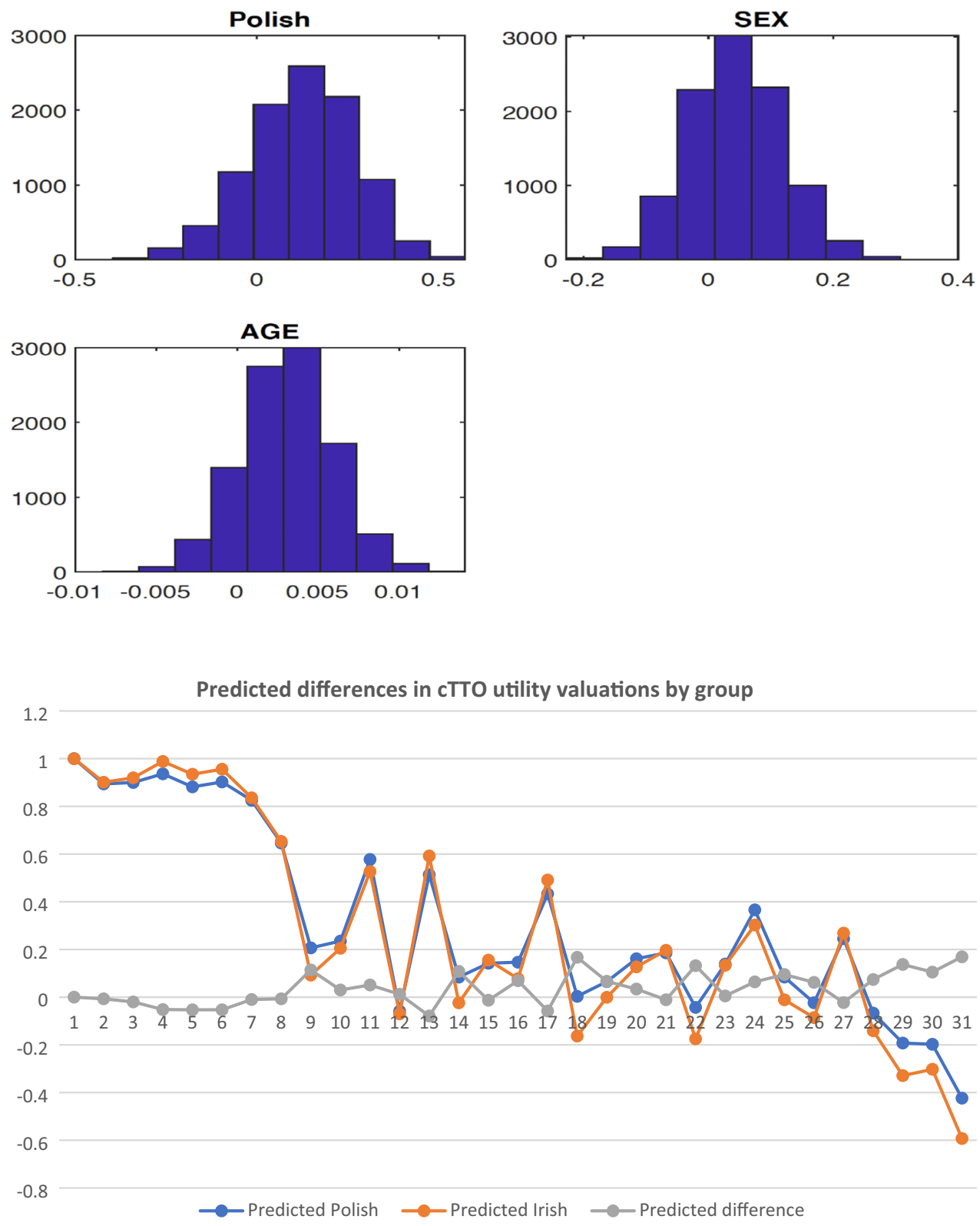

above. It is worth noting that other factors may influence a migrant's health preferences, such as their rate of time preference (discount rate), marital status and whether an individual lives in an urban household location [18, 41]. These were not controlled for in this analysis. Kelleher et al. [18] also noted other factors that were found to not be associated with migrant health preferences, which included the length of time a migrant was resident in a host country as well as income levels.

For the first time, this paper presented a novel use of a smaller design EQ-5D-5L valuation study and targeted sampling that can be used to examine the health preferences of minority subgroups. Moreover, the empirical methods of this paper add to the literature as it is the first time the use of a Bayesian statistical nonparametric model was used to estimate health preferences, coupled with a novel application of a smaller design EQ-5D-5L valuation study. For this research, the subgroups under investigation were Polish migrants and native Irish. There is a noted lack of reliable data on migrant health in the literature [42-44]. Collecting migrant health data can pose significant challenges to researchers and healthcare systems for various reasons. For example, being highly mobile groups and hard to locate, migrants can have low response rates to surveys, and data samples uncovered are often highly heterogeneous [45]. These issues may be common to those that arise with other hard-to-reach groups, such as independent young adults. The use of a smaller design EQ-5D-5L valuation study and targeted sampling can help uncover and examine migrant or other subgroups' health preferences, which can otherwise 
be hard to uncover when using the secondary analysis of national valuation studies. The samples collected on the Polish migrants and native Irish emerged unbalance across some sociodemographic characteristics, as described above. More care could have been taken to try to ensure that the data collected on the Polish migrants and native Irish were balanced. Retrospectively, this could have been achieved by collecting data on each group by age group and sex, for example. However, this extra step in the data collection would have made the data collection process more complex.

We argued that health preferences could be a predictor of an individual's valuation of health, as individuals do not directly value health when they place a utility valuation on a compromised EQ-5D-5L health state that incorporates the time spent in, and quality of, the state. The above-cited papers [14-16] have identified how one values health as an influential factor in determining an individual's healthcare use and health-related behaviours. By Polish migrants valuing severe health states higher than the native Irish, this may see Polish migrants having different healthcareseeking practices with different use and need of certain healthcare services. It is conceivable that if Polish migrants value health differently from the Irish resident population, or if any migrant group values health differently from the country's host population in which they reside, they may engage with healthcare services differently; for example, preventive care uptake. Carrieri and Bilger [14] found that those who place a high value on health are more likely to avail of preventive care than those who do not. The most evident differences in the healthcare use of migrants and host populations is in regard to their respective use of preventive care, with migrants having lower use than host populations $[5,6]$. How Polish migrants and native Irish differ in their health preferences and valuation of health may be a factor underpinning some of the disparities in preventive care uptake among migrants and host populations in Europe, as mentioned above.

Kelleher et al. [46] found that health preferences are associated with preventive care uptake. Preventive care uptake and health preferences are both underpinned by an individual's rate of time preference [18]. Therefore, by the Polish migrants and native Irish having potentially different preferences for health, this may be a factor that can contribute to some of the differences in the preventive care use between migrants and natives. Other factors can also contribute to such differences, such as the formal and informal barriers to healthcare faced by migrants [5].

More research is needed to determine how differences in health preferences, and ultimately differences in the valuation of health, can be linked to differences in healthcare utilisation and health-related behaviours among migrants and host populations. This could be done by collecting health preference data and healthcare utilisation data on a larger sample size of Polish migrants and native Irish or any migrant and native groups. Health preferences could be entered into a model as an independent variable examining its association with a range of healthcare services as used by Kelleher et al. [46]. We believe this paper is an initial building block to examining a potentially critical factor underpinning some of the disparities in healthcare utilisation and health-related behaviours among migrants and host populations.

There are some research limitations to our study that must be noted. Our model did not control for some factors that may determine an individual's health preferences, such as their rate of time preference, and this is a limitation to the research. We used snowball sampling, therefore a representative sample of Polish migrants or native Irish was not used, given the difficulties associated with trying to obtain a representative sample. When studying migrants, it can be hard to generalise the findings given the heterogeneous nature of migrant samples [45]. The findings from this study cannot be viewed as representative of Polish migrants in Ireland or in general since we did not use a representative sample. Since our survey questions were carried out in English, our sample may be underrepresentative of Polish migrants in Ireland who are not fluent in English. Our sample size was small, however we believe using the Bayesian statistical nonparametric model allowed us to make use of the small number of observations. This particular method is noted to be useful when examining differences in health state utility valuations among subgroups with limited sample sizes $[33,35$, 36]. Further research might compare the Bayesian statistical nonparametric model results with parametric methods and extend the number of subgroups under investigation. Further research could also look to compare the health preferences of various other migrant groups and host populations across Europe, especially migrant groups who have recently arrived in a host country.

The results from our study should be viewed as an assessment of the association between Polish migrant status and EQ-5D-5L health preference, and not as representations of value sets for each group more broadly, which was not the intention of this study. Polish and Irish nationals are shown to have different health preferences from their respective national valuation studies [18, 22, 47]. The results from this paper highlight that Polish migrants and native Irish differ in their health preferences for, and valuation of, severe health states. Policymakers charged with the design and implementation of migrant health policy in Ireland and Europe may find the results of this study of relevance. We have shown that migrants can value certain health states differently than host populations, which can provide an understanding of a potential new factor underpinning some of the disparities in healthcare utilisation and health-related behaviours among migrants and host populations in Europe. 


\section{Conclusion}

This study has shown that by using a novel application of a smaller design EQ-5D-5L valuation study coupled with the EQ-PVT data collection software and a Bayesian analytic design, differences in health preferences exist between a sample of Polish migrants and native Irish regarding severe health states. If replicated in other contexts, these findings may contribute to our understanding of migrant health outcomes, health-related behaviours, and healthcare utilisation, as migrants may value health differently than host populations. This study also provides proof of principle for using a smaller design EQ-5D-5L valuation study to explore differences in health preferences among other migrant or minority subgroups, which can otherwise be hard to uncover when using the secondary analysis of national EQ-5D-5L valuation studies.

Supplementary Information The online version contains supplementary material available at https://doi.org/10.1007/s41669-021-00314-2.

Acknowledgements The authors would like to acknowledge the funding and support received from the Hardiman Research Scholarship 2017 awarded by NUI Galway to Dan Kelleher, which funded this study. The authors would also like to acknowledge the support and advice from the EuroQol Research Foundation who made the survey possible, especially from Dr Kristina Ludwig and Dr Elly Stolk.

\section{Declarations}

Conflicts of interest Dan Kelleher, Samer Kharroubi, Edel Doherty, Gianluca Baio, and Ciaran O'Neill have no conflicting interests, monetary or otherwise, that would in any way inhibit the submission and publication of this research. None of the authors have any research or professional conflicts of interest to report.

Funding This study was funded by the Hardiman Research Scholarship 2017 awarded by NUI Galway to Dan Kelleher.

Ethical approval Ethical approval for this study was granted by NUI Galway's Research Ethics Committee (application number 18-Mar-13).

Consent to participate All participants consented to participate in this research and signed a consent conform.

Consent for publication (from patients/participants) All participants consented to participate in this research and its publication and signed a consent form.

Availability of data and material Can be made available upon reasonable request.

Code availability Can be made available upon reasonable request.

Author contributions DK: data curation, data modelling, writing the original draft of the paper and redrafting of the paper. SK: data modelling and redrafting of the paper. ED: redrafting of the paper and super- vision. GB: redrafting of the paper. CON: redrafting of the paper and supervision. All authors contributed fairly to this paper.

Open Access This article is licensed under a Creative Commons Attribution-NonCommercial 4.0 International License, which permits any non-commercial use, sharing, adaptation, distribution and reproduction in any medium or format, as long as you give appropriate credit to the original author(s) and the source, provide a link to the Creative Commons licence, and indicate if changes were made. The images or other third party material in this article are included in the article's Creative Commons licence, unless indicated otherwise in a credit line to the material. If material is not included in the article's Creative Commons licence and your intended use is not permitted by statutory regulation or exceeds the permitted use, you will need to obtain permission directly from the copyright holder. To view a copy of this licence, visit http://creativecommons.org/licenses/by-nc/4.0/.

\section{References}

1. European Migration Network. Migrant population and immigration statistics in EU Member States. European Migration Network; 2021.

2. JI Anton BR Munoz de 2010 Health care utilisation and immigration in Spain Eur J Health Econ. 11548798

3. G Mohan 2021 The influence of caregiver's migration status on child's use of healthcare services: evidence from Ireland Sociol Health Illn 433557574

4. A Lebano 2020 Migrants' and refugees' health status and healthcare in Europe: a scoping literature review BMC Public Health 20 1122

5. M Norredam SS Nielsen A Krasnik 2010 Migrants' utilization of somatic healthcare services in Europe-a systematic review Eur J Public Health 205555563

6. V Graetz 2017 Utilization of health care services by migrants in Europe-a systematic literature review Br Med Bull 1211518

7. M Guillon M Celse PY Geoffard 2018 Economic and public health consequences of delayed access to medical care for migrants living with HIV in France Eur J Health Econ 193327340

8. Z Kabir 2008 Smoking characteristics of Polish immigrants in Dublin BMC Public Health 8117

9. AF Constant 2018 A "healthy immigrant effect" or a "sick immigrant effect"? Selection and policies matter Eur J Health Econ 19 1103121

10. J Detollenaere S Baert S Willems 2018 Association between cultural distance and migrant self-rated health Eur J Health Econ 19 2257266

11. M Ward M Kristiansen K Sørensen 2019 Migrant health literacy in the European Union: a systematic literature review Health Educ J 7818195

12. D Sime 2014 'I think that Polish doctors are better': newly arrived migrant children and their parents' experiences and views of health services in Scotland Health Place 308693

13. S Stan 2015 Transnational healthcare practices of Romanian migrants in Ireland: inequalities of access and the privatisation of healthcare services in Europe Soc Sci Med 124346355

14. V Carrieri M Bilger 2013 Preventive care: underused even when free. Is there something else at work? Appl Econ. 452239253

15. JM Walsh SJ McPhee 1992 A systems model of clinical preventive care: an analysis of factors influencing patient and physician Health Educ Q 192157175

16. H-J Shi K Nakamura T Takano 2004 Health values and healthinformation-seeking in relation to positive change of health practice among middle-aged urban men Prev Med 39611641171 
17. A Hobbins 2020 Do people with private health insurance attach a higher value to health than those without insurance? Results from an EQ-5D-5 L valuation study in Ireland Health Policy 1246639 646

18. D Kelleher 2020 Examining the transnational health preferences of a group of Eastern European migrants relative to a European host population using the EQ-5D-5L Soc Sci Med. 246112801

19. Central Statistics Office 2021 Population and migration estimates 2021 Central Statistics Office Cork

20. NJ Devlin 2018 Valuing health-related quality of life: an EQ5D-5L value set for England Health Econ 271722

21. K Ludwig J-M Graf von der Schulenburg W Greiner 2018 German value set for the EQ-5D-5L PharmacoEconomics. 366663674

22. A Hobbins 2018 Utility values for health states in Ireland: a value set for the EQ-5D-5L Pharmacoeconomics 361113451353

23. CE Jensen 2021 The Danish EQ-5D-5L value set: a hybrid model using cTTO and DCE data Appl Health Econ Health Policy 194 579591

24. K Wang 2020 Using eye-tracking technology with older people in memory clinics to investigate the impact of mild cognitive impairment on choices for EQ-5D-5L health states preferences Appl Health Econ Health Policy 191111121

25. AS Pickard R Tawk JW Shaw 2013 The effect of chronic conditions on stated preferences for health Eur J Health Econ 144697 702

26. JW Shaw 2007 Racial/ethnic differences in preferences for the EQ-5D health states: results from the U.S. valuation study J Clin Epidemiol. 605479490

27. L Barry 2018 Euthanasia, religiosity and the valuation of health states: results from an Irish EQ5D5L valuation study and their implications for anchor values Health Qual Life Outcomes 161 152

28. M Sculpher A Gafni 2001 Recognizing diversity in public preferences: the use of preference sub-groups in cost-effectiveness analysis Health Econ 104317324

29. Z Yang 2019 Effect of health state sampling methods on model predictions of EQ-5D-5L values: small designs can suffice Value Health. 2213844

30. Z Yang 2019 Toward a smaller design for EQ-5D-5L valuation studies Value Health. 221112951302

31. M Oppe 2016 EuroQol protocols for time trade-off valuation of health outcomes Pharmacoeconomics 34109931004

32. AG Welie 2020 Valuing health state: an EQ-5D-5L value set for Ethiopians Value Health Regional Issues. 22714

33. S Kharroubi JE Brazier A O'Hagan 2007 Modelling covariates for the SF-6D standard gamble health state preference data using a nonparametric Bayesian method Soc Sci Med 64612421252
34. S Kharroubi A O'Hagan JE Brazier 2005 Estimating utilities from individual health preference data: a nonparametric Bayesian method J Roy Stat Soc Ser C (Appl Stat) 545879895

35. SA Kharroubi JE Brazier S McGhee 2014 A Comparison of Hong Kong and United Kingdom SF-6D health states valuations using a nonparametric Bayesian method Value Health. 174397405

36. SA Kharroubi A O'Hagan JE Brazier 2010 A comparison of United States and United Kingdom EQ-5D health states valuations using a nonparametric Bayesian method Stat Med 2915 16221634

37. E Stolk 2019 Overview, update, and lessons learned from the International EQ-5D-5L valuation work: version 2 of the EQ5D-5L valuation protocol Value Health. 2212330

38. A Hobbins 2018 The health of the residents of Ireland: population norms for Ireland based on the EQ-5D-5L descriptive system-a cross sectional study HRB Open Res. 122

39. EB Henry 2020 Estimation of an instrument-defined minimally important difference in EQ-5D-5L Index Scores based on scoring algorithms derived using the EQ-VT version 2 valuation protocols Value Health. 237936944

40. C Quintal 2012 Aversion to geographic inequality and geographic variation in preferences in the context of healthcare Appl Health Econ Health Policy 72121136

41. FA Sayah 2016 Determinants of time trade-off valuations for EQ5D-5L health states: data from the Canadian EQ-5D-5L valuation study Qual Life Res 25716791685

42. C Straßner 2017 Quality circles to identify barriers, facilitating factors, and solutions for high-quality primary care for asylum seekers BJGP Open. https://doi.org/10.3399/bjgpopen17X101133

43. AW Smalen de 2021 Developing an evidence assessment framework and appraising the academic literature on migrant health in Malaysia: a scoping review BMJ Open. 111 e041379

44. M Wallace B Wilson 2021 Age variations and population overcoverage: is low mortality among migrants merely a data artefact? Popul Stud. https://doi.org/10.1080/00324728.2021.1877331

45. P Mladovsky 2009 A framework for analysing migrant health policies in Europe Health Policy 9315563

46. D Kelleher E Doherty C O'Neill 2021 Health preferences and preventive care utilisation: how EQ-5D-5L health preferences may affect uptake Prevent Med Rep. 24101514

47. D Golicki 2019 Valuation of EQ-5D-5L Health States in Poland: the First EQ-VT-Based Study in Central and Eastern Europe Pharmacoeconomics 37911651176

\section{Authors and Affiliations}

\section{Dan Kelleher ${ }^{5}$ (D) Samer Kharroubi ${ }^{2} \cdot$ Edel Doherty $^{1} \cdot$ Gianluca Baio $^{3} \cdot$ Ciaran O'Neill $^{4}$}

Samer Kharroubi

sk157@aub.edu.lb

Edel Doherty

Edel.doherty@nuigalway.ie

Gianluca Baio

g.baio@ucl.ac.uk

Ciaran O'Neill

Ciaran.oneill@qub.ac.uk

1 Health Economics and Policy Analysis Centre, The Institute for Lifecourse and Society, Upper Newcastle, NUI Galway, National University of Ireland, Galway H91C7DK, Ireland
2 Department of Nutrition and Food Sciences, Faculty of Agricultural and Food Sciences, American University of Beirut, Riad El Solh, PO Box 11-0236, Beirut, Lebanon

3 Department of Statistical Science, University College London, Gower Street, London WC1E 6BT, UK

4 Institute of Clinical Sciences, Centre for Public Health, Queen's University Belfast, Royal Victoria Hospital, Block B, Belfast BT12 6BA, Northern Ireland, UK

5 The Institute for Lifecourse and Society, NUI Galway, Room 2007, Upper Newcastle, Galway H91C7DK, Ireland 